\title{
Physical activity, transportation mode and sedentary time on the development of overweight and obesity in school-age children in China: China Health and Nutrition Survey (CHNS) 2010-2012
}

\author{
GuYuan Tang ${ }^{1}$, PuiYee Tan ${ }^{1}$, Qian Zhang ${ }^{2}$, JunSheng Huo ${ }^{2}$ and YunYun Gong ${ }^{1}$ \\ ${ }^{1}$ School of Food Science and Nutrition, Faculty of Environment, University of Leeds, Leeds LS2 9JT, United Kingdom \\ and \\ ${ }^{2}$ National Institute for Nutrition and Health (NINH), Chinese Center for Disease Control and Prevention \\ (China $C D C$ ), Beijing
}

Worldwide prevalence of childhood obesity has increased substantially in the past decades and was associated with significant impact on child's physical and social development. High energy-dense foods and physically inactive are the major lifestyle risk factors for childhood obesity ${ }^{(1)}$. Therefore, this study aimed to determine the prevalence of overweight and obesity among the Chinese schoolaged children, and the relationships between childhood obesity and lifestyle factors, particularly on physical activity and dietary intake.

Data for this study was obtained from Chinese Health and Nutrition Survey (CHNS) 2010-2012 ${ }^{(2)}$. Social-demographic and physical activity data were collected using structured questionnaires. Dietary intake was assessed using a 100-item FFQ. Overweight and obesity were defined as BMI at 85 th-95thand $>95$ th percentiles, respectively, specified for age and gender ${ }^{(3)}$. 24,722 children aged 6-17 years old from 31 provinces were recruited using a multistage random-cluster sampling process 2 . Of those, 2,617 participants who had complete data on the aforementioned parameters were included in this study. Statistics: Multivariate logistic regression analysis was performed to determine the associations between lifestyle factors and the odds of overweight and obesity. Mann-Whitney test was performed to assess the difference in average daily food consumption between the low and high physical activity groups.

$16.3 \%$ of our study participants were found to be overweight, and of those, $7.3 \%$ were obese. Children using motorized transportation method (including car, bus, taxi or other public transport) to school and who spent $\geq 3 \mathrm{~h} /$ day of sedentary activity were associated with increased odds of overweight and obesity $(\mathrm{OR}=1.296, \mathrm{CI}=1.030-1.631 ; \mathrm{p}=0.027$ and $\mathrm{OR}=1.485, \mathrm{CI}=1.020-2.164 ; \mathrm{p}=$ 0.039 , respectively) compared to non-motorized transportation and $<2 \mathrm{~h} /$ day of sedentary activity, after adjusting for covariates gender, age, income level, resident type, and total energy intake. Interestingly, children who spent $\geq 60 \mathrm{mins} /$ day of physical activity were significantly associated with increased odds of overweight and obesity $(\mathrm{OR}=1.472, \mathrm{CI}=1.185-1.828 ; \mathrm{p}<0.001)$ compared to $<60 \mathrm{mins} / \mathrm{day}$. This may be explained by the increase in average daily consumption of fish, eggs, dairy products, fruits, vegetables, legumes, snacks, and beverages, in the physically active group $(\geq 60 \mathrm{mins} /$ day $)(\mathrm{p}<0.05)$. Moreover, $\geq 41.5 \mathrm{~g} / \mathrm{day}$ of egg, and $\geq 113.3 \mathrm{~g} /$ day of dairy product consumption were associated with increased odds of overweight and obesity $(\mathrm{OR}=1.645, \mathrm{CI}=$ $1.119-2.417 ; \mathrm{p}=0.011$ and $\mathrm{OR}=1.432, \mathrm{CI}=1.000-2.053 ; \mathrm{p}=0.050$, respectively), compared to lower consumption. However, such associations did not reach statistical significance after adjusting for covariates $(\mathrm{p}>0.05)$. No other dietary factor alone was found significantly associated with overweight and obesity.

Motorised transportation to school and sedentary time were significantly associated with increased risk of childhood overweight and obesity. Similar finding was found in physical activity; however, this may be due to the increase in total energy intake associated with higher physical activity level. Dietary factor alone was not found to be significantly associated with overweight and obesity.

\section{Acknowledgements}

The authors are grateful to Professor Junsheng Huo and Professor Jing Sun from Chinese Centre for Disease Control and Prevention's (CDC) National institute of Health and Nutrition (NINH) for providing the CHNS 2010-2012 data. The authors acknowledge the funding support from the Biotechnology and Biological Sciences Research Council (BBSRC) (Grant number: BB/T008989/1) through Global Challenge Research Fund (GCRF) 2020 granted to the University of Leeds project titled "Addressing micronutrient deficiencies associated with the double burden of childhood malnutrition in China, a combined food system framework".

\section{References}

1. Mistry SK \& Puthussery S (2015) Public Health 129, 200-209

2. Popkin BM, Du S, Zhai F et al. (2010) Int J Epidemiol 39, 1435-1440

3. Group of China Obesity Task Force (2004) Zhonghua Liu Xing Bing Xue Za Zhi 25, 97-02. 